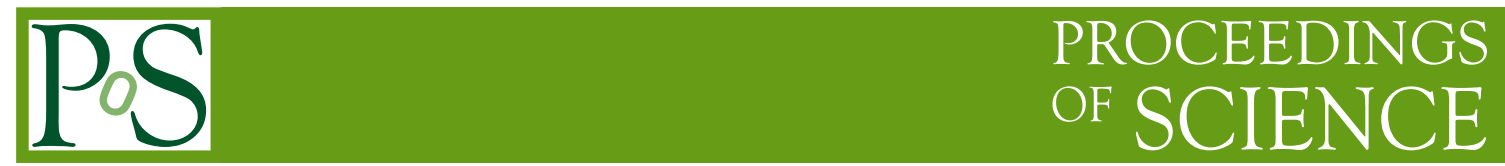

\title{
Cooling: Experience from ATLAS \& CMS
}

\author{
Koichi Nagai ${ }^{* i}$ \\ on behalf of the ATLAS and CMS Collaborations \\ $B N L$ \\ E-mail: Koichi.Nagai@cern.ch
}

The cooling system of a large scale silicon detector system is critical for the long term operation of detectors in the harsh radiation environment of the LHC. The ATLAS and CMS experiments have developed and commissioned their large and complex distributed systems over the last few years. The systems are now complete and are in operation. In this publication I will describe the ATLAS and CMS systems and summarise some of the experience gained in the development and operation of these systems.

VERTEX 2009 (18th workshop)

September 13-18 2009

VELUWE, the Netherlands

*Speaker.

${ }^{\dagger}$ Currently at University of Tsukuba. 


\section{Introduction}

Silicon technology is widely used in high energy physics experiments to measure the trajectories, momenta and vertices of charged particles close to the interaction point. The ATLAS and CMS experiments at the Large Hadron Collider (LHC) at CERN have chosen a similar detector layout for their innermost tracking detectors: pixel detectors at the inner most layers surrounded by silicon strip detectors. Since the detectors are placed close to the beam pipe, they are irradiated by a large flux of particles during LHC operation. This radiation induces several types of damage to the silicon sensors throughout the lifetime of the experiment. The most important consequences of the radiation for a long term operation are the increase depletion voltage and leakage currents in sensors, which could lead, if uncontrolled, to thermal runaway. Studies on radiation damage show that these effects can be minimised by keeping the silicon sensors at or below $0^{\circ} \mathrm{C}$ during both the beam-on and beam-off (maintenance) time. The cooling systems for semiconductor detectors are recognised as one of the key components for the long term detector operation of these detectors.

\section{Cooling systems}

\subsection{Requirement on cooling system}

To maintain silicon sensor's temperature at or below $0^{\circ} \mathrm{C}$, it is necessary to maintain the temperature of the cooling staves below $-20^{\circ} \mathrm{C}$ due to the limited thermal transfer efficiency between cooling staves and sensors. It's not difficult at all to realise the required temperatures by using industrial freezer technology. There are, however, many mechanical, electrical and thermal requirements placed on tracking detectors located at the centre of particle physics experiments that are not normal requirements in the refrigeration industry.

- Low radiation length and small physical size of cooling staves

- The fluid must be non-corrosive and non-flammable

- Reliability for a long term non-stop operation with minimal or zero maintenance periods

- Minimal over-pressure on detector cooling staves

- Both experiments estimate the total power consumption of semiconductor detectors of approximately $56 \mathrm{~kW}$ after full irradiation.

Taking these requirements into account, the cooling system becomes a large, complicated and distributed.

\subsection{CMS cooling system}

CMS have selected a mono-phase cooling system. The mono-phase cooling has been used in many high energy physics experiments and is an established technique. The system consists of three parts as shown in Figure 1: the chiller group which is a commercial system; the detector cooling units which circulate $\mathrm{C}_{6} \mathrm{~F}_{14}$ to the detectors; and the brine group which is a heat transfer line between the chiller and detector cooling units using $\mathrm{C}_{6} \mathrm{~F}_{14}$. The brine group is isolated from 


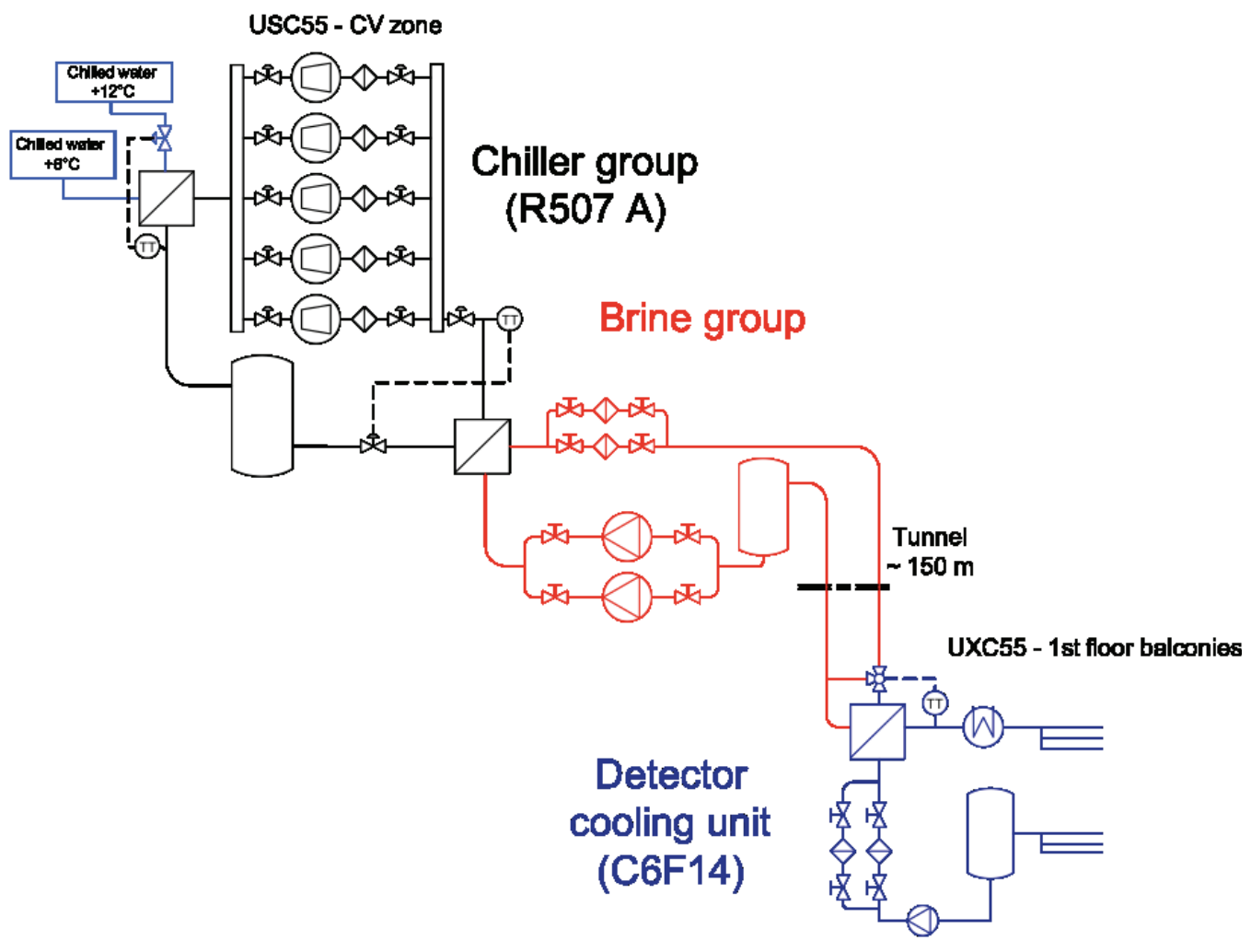

Figure 1: Schematic view of the CMS cooling system.

the chiller and cooling units by heat exchangers. Following a failure in heat exchangers, the brine in the system was replaced by $\mathrm{C}_{6} \mathrm{~F}_{14}$. Each sub-tracker system has an independent cooling unit which controls operation temperature for each sub-tracker separately. The system circulates the liquid to 198 cooling circuits for strip and pixel detectors with a total flow rate of 18 litres/sec. Inside detector volumes cooling circuit are connected to several branches.

\subsection{ATLAS cooling system}

ATLAS has chosen an evaporative (two phase) cooling system with $\mathrm{C}_{3} \mathrm{~F}_{8}$ rather than monophase cooling for the following reasons:

- Larger cooling capacity per unit volume due to the utilisation of the latent heat of evaporation rather than the specific heat capacity.

- Higher heat transfer coefficient

The above features drive the design to lower mass flow and smaller size for cooling circuits resulting in a lower radiation length of the liquid itself and the material of the cooling staves. The system itself consists of a cooling plant, distribution racks and detector cooling circuits as shown in Figure 2. A total of 204 circuits are divided into four quadrants in the $r-\phi$ plane. Each quadrant has a distribution rack which receives fluid from the plant and distributes it to each circuit. The schematic view of one cooling circuit is shown in Figure 3. Each circuit has a pressure (PR) and a 


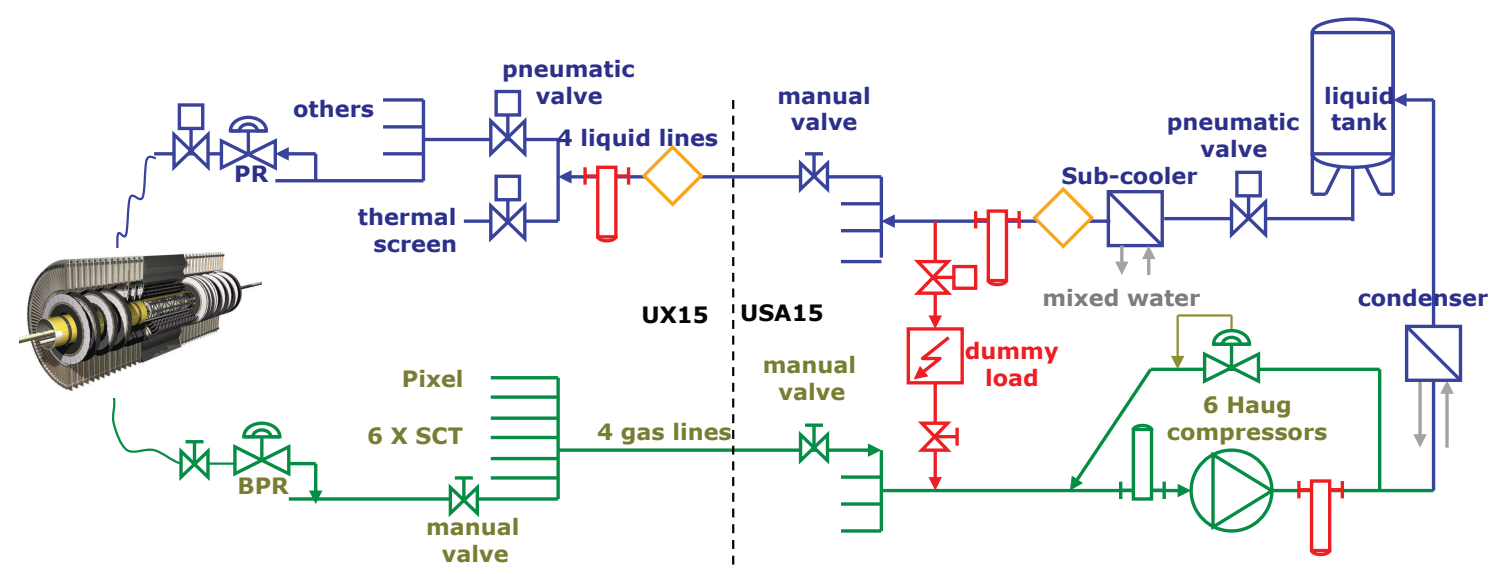

Figure 2: The schematic of ATLAS evaporative cooling system.

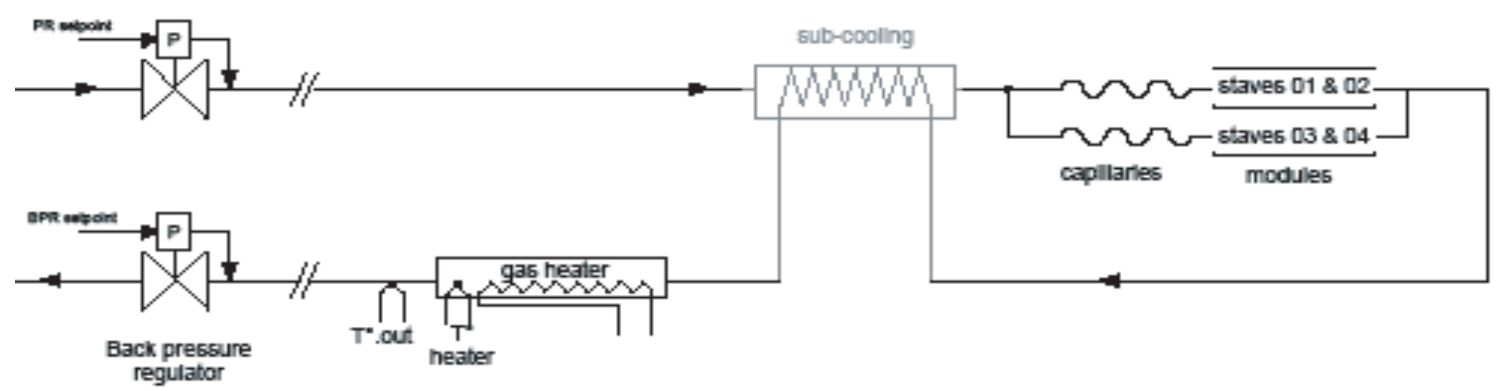

Figure 3: The schematic of one cooling circuit in ATLAS evaporative cooling system.

back pressure regulator (BPR) in the distribution rack to control liquid and vapour pressure, respectively. The BPR defines the evaporation temperature. The over-saturated fluid is piped to the Inner Detector (ID) volume about $30 \mathrm{~m}$ away from the distribution rack. There the incoming liquid is cooled by the outgoing vapour in a counter-flow heat exchanger (HEX) which acts as a sub-cooler and minimises evaporation before the fluid reaches at the detector cooling stave. Then the circuit connects to 1, 2 or 3 detector staves by capillaries depending on the particular detector configuration. A capillary, with a prefixed length, is installed at the entrance of each detector cooling stave, and defines the mass flow according to pressure drop across it. The detector cooling stave was designed to transfer heat efficiently from detector modules to the cooling fluid by thin wall pipes. The fluid starts to evaporate at the end of capillaries and cools down the stave and incoming fluid. The rest of fluid is evaporated at the end of the ID volume and warmed up to room temperature by high power density heaters which were installed in limited space inside the hermetically controlled tracker volume and are controlled by a Programmable Logic Control (PLC) based system according to the vapour temperature at down-stream. Temperatures on the heater body are also monitored by an interlock system which stop heater power in case of high temperature. The main part of the plant is installed in the USA15 side cavern where there are a total of 7 HAUG compressors (the last one installed in 2009) and a control system built by three PLC units. Two PLCs are dedicated to control 204 cooling circuits and their heaters according to the temperature at the exit of the ID 

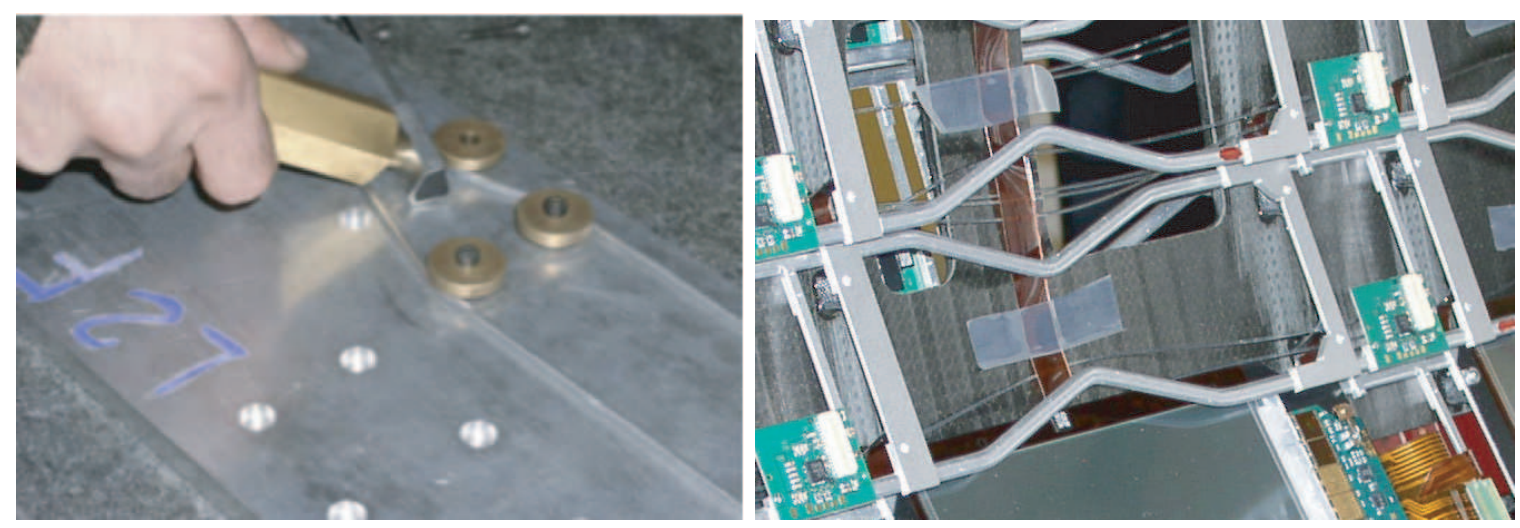

Figure 4: Construction of cooling staves in CMS. Left: The jig to shape the staves. Right: Assembled cooling staves.

volume and the other turns on/off compressors according to the load. One compressor has the ability to fine-tune the compression capacity using a bypass line. The system communicates through an additional PLC with the detector control system.

\section{Construction and installation of the cooling systems}

The construction of the cooling system is a very delicate task, especially close to the silicon detectors, where low radiation length is required and the parts are necessarily low mass and hence fragile. Many pieces were produced manually by skilled engineers and technicians. Figure 4 shows a handmade stave for the CMS detector and its jig. After the assembly of such delicate pieces, it is very important to perform a significant number of quality assurance (QA) tests. Any defects not caught by poor QA would cause problems in the later stage of the installation and operation. ATLAS found poor soldering connections on the HEX for the SCT End-Cap (SCT-EC) during the installation, as shown in Figure 5. All SCT-EC HEX were examined by X-ray and repaired if necessary.

\section{Commissioning}

\subsection{CMS}

CMS started commissioning in 2008 after lowering the detector into the cavern. The commissioning continued for 7 months. The leak rate of the cooling fluid from the detector cooling units increased to $50 \mathrm{~kg} / \mathrm{day}$ by the end of the commissioning period. Their leak hunting investigations found many leaks at multiple locations. The leaks appeared for several reasons: the use of valves or resin in the connector which were not compatible with $\mathrm{C}_{6} \mathrm{~F}_{14}$, the location of connections did not allow proper closing of the fittings and in some cases the poor quality of the fitting and plastic pipes. Since those problems were seen on so many cooling units the CMS collaboration decided to refurbish all cooling units at the beginning of 2009. The scope of the refurbishment was:

- to replace the connection by brazing or welding and the use of proper material. 


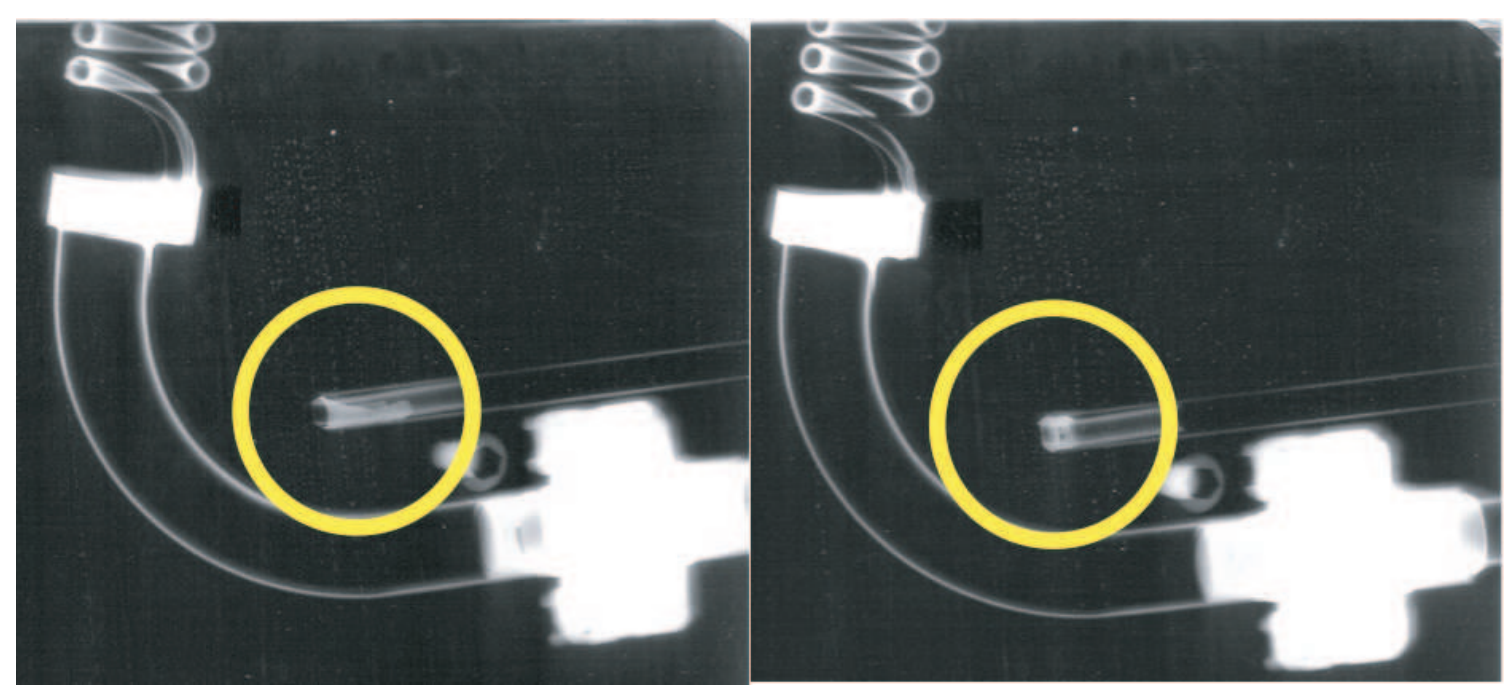

Figure 5: The X-ray shows a poorly soldered connection (in circle) on HEX for SCT-EC in ATLAS which caused a problem during the installation.

- the use of the highest quality components and to implement a very thorough QA plan including visual inspection and X-ray imaging of at least $10 \%$ of the welds, as well as to perform leak and pressure tests at every stage of the assembly process.

In parallel, a leak test was done on the detector circuits to check the leak rate which became a reference point for later leak tests. In addition to the leak problems some detects of the system, such as the inability to purge the gas from the system, were remedied at the same time. They also found that it was necessary to improve the PLC controls and to have some additional instrumentation to monitor critical components in order to better understand the status of the system during operation. The cooling system was restarted in June 2009. The leak rate improved as shown in Figure 6.

\subsection{ATLAS}

The cooling plant was partially installed in June 2006. As soon as one quarter of the ATLAS evaporative cooling system became available (and before the installation of the inner detector), a pilot test was performed with 5 circuits including HEX, heater, capillaries and dummy heat source. The results of these tests with the real cooling system but without the detector cooling staves revealed two problems. The first was the regulation of the heater power which in some cases could not be stabilised. The problem occurred close to a change in a pipe diameter which was in turn close to the location of the regulation sensor, where the non-evaporated liquid was mixed with hot vapour and cooled the whole area. Since the temperature regulation sensors were installed in this area, this phenomena caused instabilities in the regulation procedure. The solution was to move the sensors further down-stream where the fluid temperature is stable. The other problem is that the BPR could not achieve the low back pressure value which is necessary to achieve $-25^{\circ} \mathrm{C}$. The investigation showed that the stainless steel membrane was too rigid to regulate at low pressure. All membrane were replaced by Gylon. The BPR were tested in situ using a test circuit which can provide a defined mass flow for cooling circuits. Figure 7 shows the difference between the dome control and the supply pressures. In February 2007, one of the evaporative heaters developed an 


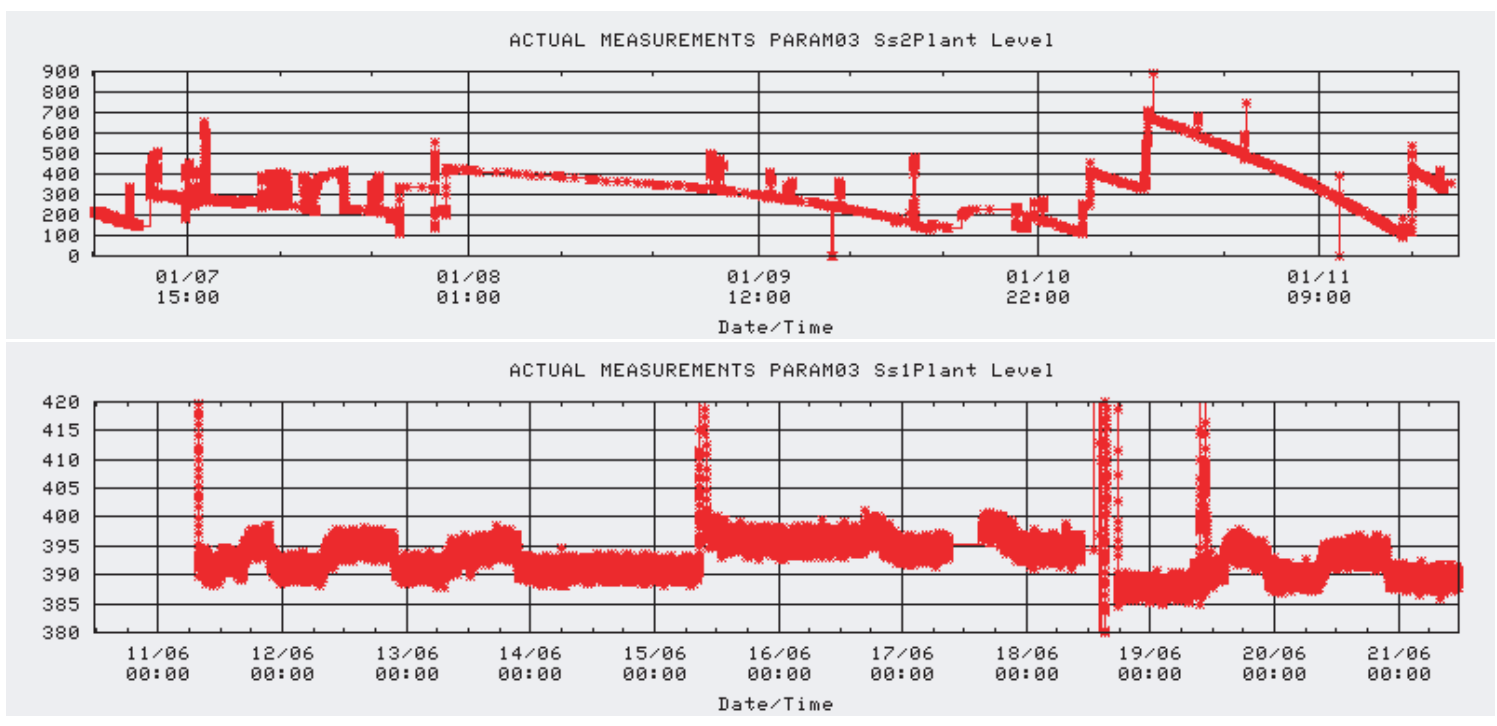

Figure 6: CMS tank level of $\mathrm{C}_{6} \mathrm{~F}_{14}$ in 2008 (top) and in 2009 (bottom). A big leak was developed in 2008. Their effort to fix reduced the leak rate in 2009 successfully.

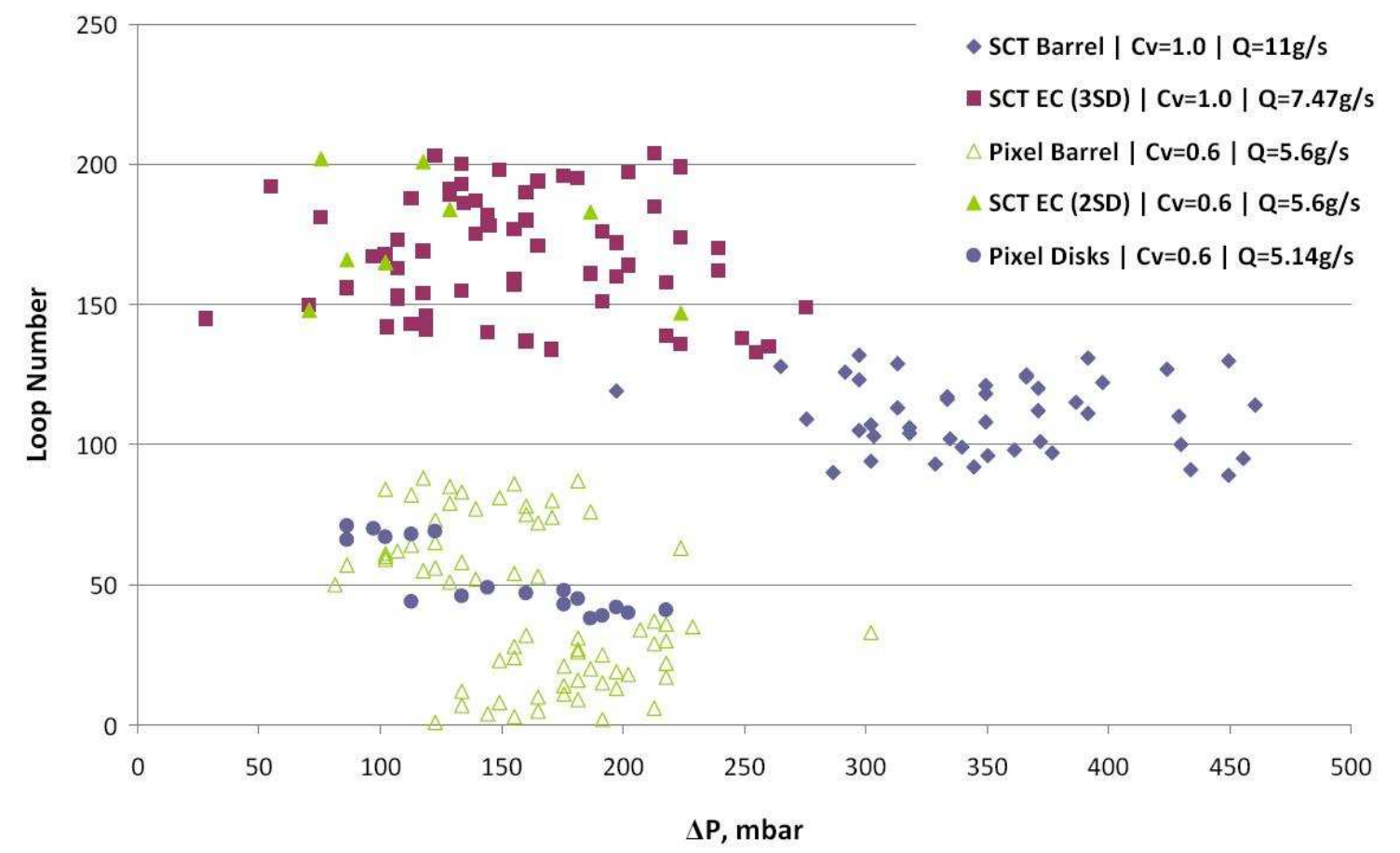

Figure 7: The difference between dome and supply pressures for each cooling circuit with the Gaylon membrane measured with a test circuit at the distribution racks in ATLAS. 
electrical short to ground in the splice union of one of the power line connectors. This electrical failure also revealed that the heater power supply control system couldn't stop power to the heater even though an interlock signal, designed to stop the power, was issued. The design flaw in the supply system was quickly found in the heater powering system and the supplying scheme subsequently modified. Furthermore, a new independent safety system was introduced, which will turn off the heater power racks when the heater surface temperature monitored by the interlock system, exceeds $120^{\circ} \mathrm{C}$. As for the origin of the short inside the union, after extensive tests it was concluded that it was an intrinsic problem of electrical breakdown at the interface between the epoxy sealing the connector and the mineral insulated (MI) cable caused by trapped humidity in the MI cable. Finally a new design of splice connector was supplied by the vender and this solved the problem. However, the problems had already caused significant modifications in the ID installation sequence and schedule and the overall mechanical configuration of the cooling system within the ID volume. Finally all heaters were installed in a new position after the unions were reassembled and the heaters had been mechanically modified to fit their new location.

The commissioning of the cooling system started with the SCT barrel in November 2007 before the completion of the cooling installation to confirm the modification and to advance the detector commissioning schedule. In April 2008, the installation of all cooling circuits was completed. In May 2008, during the commissioning of the Pixel detector, the system experienced a catastrophic failure of three of the HAUG compressors caused by an extended period where the magnetic bearings were slipping without driving the compressor. The brief commissioning period was however, long enough to identify another regulation problem of the Pixel heaters. Several downward pointing heaters in the bottom half of the detector were unstable with respect to PLC regulation. Additional thermocouples had been installed in the flow using a union at the end of the heater pipe which is $15-20 \mathrm{~cm}$ away from the heater and these were causing the regulation problems. Additional regulation sensors were installed further downstream. These modifications were developed and verified in a like-for-like component test setup in a building on the surface close to the ATLAS experiment.

Figure 8 shows the thermal image of the thermocouples installed in the union, with a cold spot in front of the union and a hot spot after the union. The unstable regulation was caused by the sensor position which was too close to where the residual liquid mixed with the hot vapour. The new sensor position was tested in the new test setup and new control sensors for circuits with unstable regulation were installed at further down-stream (1-2 $\mathrm{m}$ away from the heater). After the repair of the damaged compressors and cleaning of the plant, the commissioning restarted in August 2008. The commissioning of the Pixel Detector restarted with a modified heater control system. The cooling system was operated fully until the end of 2008 , achieving $96 \%$ availability during the last three months of that year. However it was found that because of excessive vibrations induced in the pipes attached to the compressors the compressor gradually developed leaks at the threaded connection and at welded junctions. The pipe work was completely redesigned and qualified by in-situ stress and vibration analysis. This modification was applied to all six compressors during the shut-down at the beginning of 2009. At the same time the distribution racks were modified to improve their reliability and to add new monitoring and control features. The complete cooling system was in operation again in June 2009. A systematic study of the operational temperatures found that the SCT Barrel circuits cannot be made to operate at their lowest specified temperatures. 


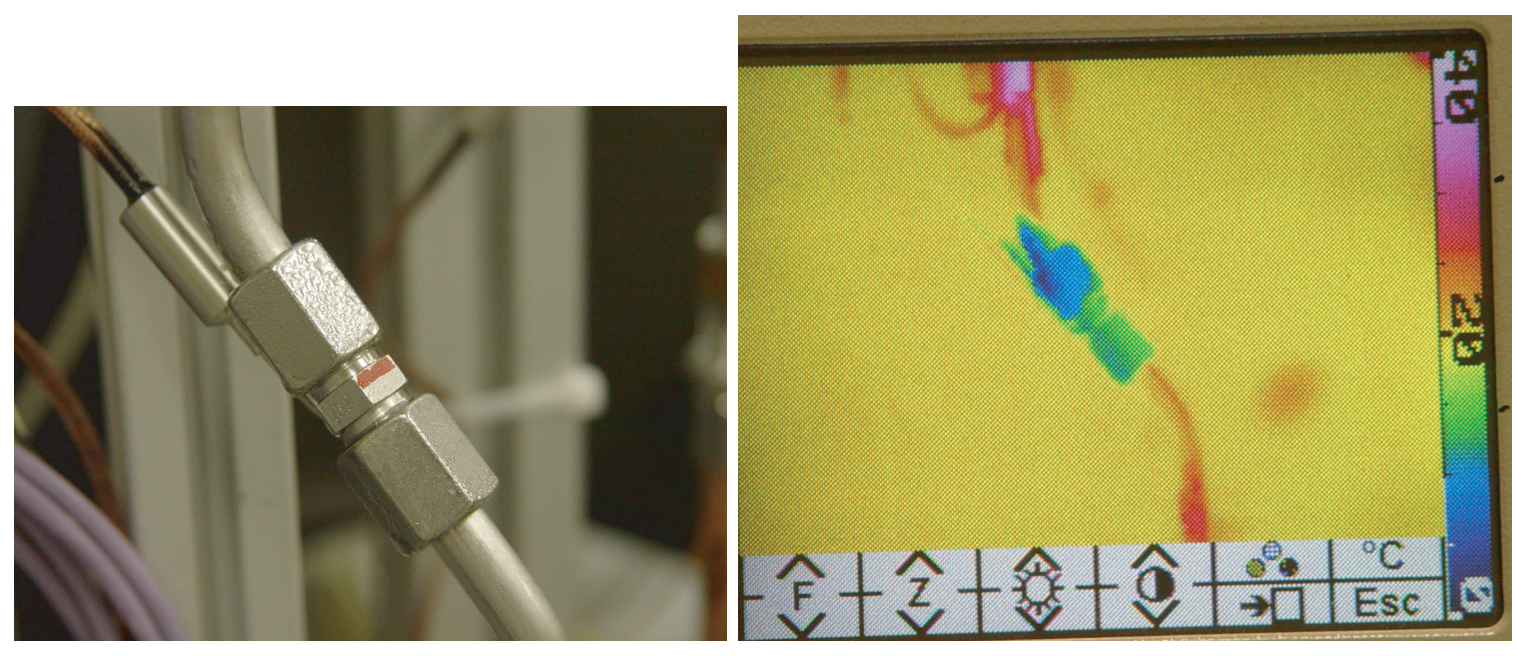

Figure 8: The heater control sensor for the ATLAS Pixel circuit. Left: The thermocouples are installed in the union at the exhaust. Right: Thermal image of the union shows a cold spot in front of the union and a hot spot after the union. The fluid flows from top to bottom.

The problem doesn't cause any adverse effect yet and will not for many years, but it will affect of leakage current at the end of the life of the detector. The problem is caused by an excessive pressure drop due to the impedance of the limited exhaust pipe diameter. Since the minimum pressure at the BPR is limited by the inlet pressure of the compressors which is about 0.8 bar, there are a certain limit on the amount of the pressure drop. The budget of the pressure drop for the operation at $-25^{\circ} \mathrm{C}$ where the evaporation pressure of $\mathrm{C}_{3} \mathrm{~F}_{8}$ is $1.67 \mathrm{bar}$, is approximately 0.9 bar. A test stand was built in a surface building to study the problem and the system test was refined to represent in more detail of the SCT barrel circuit including last minute modifications in situ such as changes in the routing of pipes and the position of heaters. The results from the system test reproduced the problem. Several ideas for a solution have been proposed including a thermal siphon system and a blend of two kinds of fluorocarbons. These will be studied in the near future.

\section{Conclusion}

The cooling systems for the silicon detectors in the CMS and the ATLAS experiments were developed, installed and commissioned. Both systems have delivered an excellent operation efficiency. During the commissioning many problems were found and most of them have been fixed. From this experience, it was recognised that it is very important to have good quality assurance in production and construction, and to perform an accurate system test with the final design during the development phase. The systems are now entering in phase of operation and maintenance. It will require some experience to establish the way to maintain the cooling systems together with detector operation.

\section{References}

[1] D. Attree et. al. 2008 The evaporative cooling system for the ATLAS Inner Detector, JINST 3 P07003 